\title{
SCIENTIFIC REPORTS

\section{OPEN Comparisons of lung and gluteus transcriptome profiles between yaks at different ages}

Received: 11 November 2018

Accepted: 9 September 2019

Published online: 02 October 2019

\author{
Jin-Wei Xin ${ }^{1,2}$, Zhi-Xin Chai ${ }^{3}$, Cheng-Fu Zhang ${ }^{1,2}{ }^{2}$ Qiang Zhang ${ }^{1,2}$, Yong Zhu ${ }^{1,2}$, Han-Wen Cao ${ }^{1,2}$, \\ Qiu-Mei Ji ${ }^{1,2}$ \& Jin-Cheng Zhong ${ }^{3}$
}

The yak, Bos grunniens, is the only large mammal in the Qinghai-Tibet Plateau and has been bred to provide meat, milk, and transportation. Previous studies indicate that the immune system contributes to the yak's adaptation to high-altitude environments. In order to further investigate changes in immune function during yak development, we compared the transcriptome profiles of gluteus and lung tissues among yaks at 6, 30, 60, and 90 months of age. Analyses of significantly differentially expressed genes (DEGs) in lung tissues revealed that immune function was more activated at 6-months and less activated at 90-months than in the 30 and 60-month-old animals. DEG exploration in gluteal tissues revealed that immune functions were more highly activated at both 6 and 90 -months, compared with 30 and 60-months. Immune system activation in the muscle and lung tissues of 30-month-old yaks may increase their resistance to infections, while decreased may be due to aging. Furthermore, the higher immune activation status in the gluteal tissues in 90-month-old yaks could be due to muscle injury and subsequent regeneration, which is supported by the fact that 5 unigenes related with muscle injury and 3 related to muscle regeneration displayed greater expression levels at 90 -months than at 30 and 60-months. Overall, the present study highlights the important role of the immune system in yak development, which will facilitate future investigations.

The yak Bos grunniens is an amazing species, displaying a high tolerance to low oxygen and low temperature conditions ${ }^{1}$. Compared with related species, yaks have developed powerful respiratory and circulatory systems ${ }^{2-4}$, as well as special mechanisms underlying regulating the metabolism of nitrogen in the kidney ${ }^{5,6}$. Molecular studies have revealed upregulated expression of genes that might contribute to this adaptation to high altitude environments, including genes involved in hemocytogenesis, angiogenesis, heme binding, glycerolipid biosynthesis, electron carrier activity, immunity, cytochrome oxidase activity, and muscle proliferation and contractility ${ }^{7-9}$.

As the only large mammal species living in the Qinghai-Tibet Plateau, yaks provide meat and milk to local residents, while also serving agricultural and transportation roles ${ }^{1}$. To develop better breeding practices, more knowledge on the development and growth of yaks is required. As previously reported, yak dermis thickness increases with age, which may increase tolerance to low temperature ${ }^{10}$. Adult yaks had thinner media muscles in their pulmonary arteries and a thinner blood-air barrier in the lungs than did juvenile yaks ${ }^{11}$, which would improve the animal's air-exchange efficiency. As age increases, functional thymic tissue is replaced by adipose, and connective tissues and thymic capsules thicken ${ }^{12}$, suggesting the changes in the immune system. These studies have explored morphological and histological changes during yak development. Few studies have been performed at a molecular level, however. One proteomics study revealed that proteins associated with cell adhesion, cell motility, keratinocyte differentiation, cytoskeleton organization, osteoblast differentiation, and fatty acid metabolism regulated horn development ${ }^{13}$. mRNA and protein expression levels of CD3 and S100 $\beta$ in the thymus were shown to decrease with age, whereas the expression level of caspase-3 increased with age, suggesting changes in immune function with advancing aging ${ }^{12}$. Obviously, the molecular understanding of yak development is still lacking, which is important for optimization of yak breeding practices.

\footnotetext{
${ }^{1}$ State Key Laboratory of Hulless Barley and Yak Germplasm Resources and Genetic Improvement, Lhasa, P.R. China. ${ }^{2}$ Institute of Animal Science and Veterinary, Tibet Academy of Agricultural and Animal Husbandry Sciences, Lhasa, P.R. China. ${ }^{3}$ Key Laboratory of Qinghai-Tibetan Plateau Animal Genetic Resource Reservation and Utilization, Sichuan Province and Ministry of Education, Southwest Minzu University, Chengdu, P.R. China. Jin-Wei Xin and ZhiXin Chai contributed equally. Correspondence and requests for materials should be addressed to Q.-M.J. (email: qiumei05@126.com)or J.-C.Z. (email: zhongjincheng518@126.com)
} 
Transcriptome sequencing is a powerful tool to screen changes in gene expression profiles, and has been widely used to investigate animal development ${ }^{14,15}$. Our previous study ${ }^{16}$ compared the transcriptome profiles of lung and gluteal tissues between yak, Tibetan cattle (high-altitude animals), Sanjiang cattle, and Holstein cattle (low-altitude animals). The results showed that $11 \mathrm{KEGG}$ pathways associated with innate immunity were more activated in yak and Tibetan cattle than in low-altitude cattle breeds, suggesting that immune function might be important for yak adaptation to high-altitude environments. In the present study, in order to further explore changes in immune function during yak development, gluteal and lung tissues were collected from yaks of different ages and then subjected to transcriptome sequencing. Bioinformatics analyses were carried out to identify differentially expressed genes (DEGs) and enriched pathways and real-time quantitative PCR (RT-qPCR) was applied to validate gene expression levels. The potential roles of DEGs are discussed, and our results contribute to the current understanding of the molecular mechanisms underlying immune function development in the yak.

\section{Materials and Methods}

Ethics statement. The protocol used in the present study was approved by the Institutional Animal Care and Use Committee of Southwest Minzu University (Chengdu, P. R China) and all methods were carried out in accordance with the approved guidelines. No local regulations or laws were overlooked throughout the course of the investigation. Samples used in the present study were purchased from local farmers.

Sample collection. In yak farms, breeders generally divide yaks into four age stages: juvenile $(<1$ year), youth (1-3 years), prime (3-7 years) and senior ( $>7$ years). Yaks older than 7 years are generally sacrificed for meat. Thus, in the present study, samples from juvenile (6-month old), youth (30-month old), adult (60-month old), and senior (90-month old) yaks were collected for analyses. All yaks used in this study were raised at a local farm at Keqiong Village, Kamaduo Town, Leiwuqi County, Changdu City, P. R. China $\left(96^{\circ} 22^{\prime} 45.26^{\prime \prime} \mathrm{N}\right.$, $31^{\circ} 5^{\prime} 54.6^{\prime \prime} \mathrm{E}$, altitude $\left.=4,343 \mathrm{~m}\right)$. Farmers regularly kill yaks to sell meat in the market. Between Oct $21^{\text {st }}$ and $22^{\text {nd }}$, 2017, lung and gluteal tissues were collected from healthy female animals at each stage (6 months, 30 months, 60 months, and 90 months), sacrificed at the local farm (altitude 4,343 m). Tissues were immediately frozen in liquid nitrogen. Three individuals were prepared for each age group as three biological replicates.

RNA extraction and transcriptome sequencing. Total RNA was extracted using Biozol reagent (Bioer, Hangzhou, China), and was then visualized on $1 \%$ agarose gel. The RNA quality was estimated using a NanoPhotometer ${ }^{\circledR}$ spectrophotometer (IMPLEN, CA, USA) and the Agilent Bioanalyzer 2100 system (Agilent Technologies, CA, USA). RNA samples with an RNA integrity number (RIN) higher than 8.0 were qualified. The RNA quantity was determined using the Qubit ${ }^{\circledR}$ RNA assay kit (Life Technologies, CA, USA).

In order to construct sequencing libraries, mRNA was enriched by treating total RNA with the Epicentre Ribo-zeroTM rRNA removal kit (Epicentre, USA). Sequencing libraries were constructed using NEBNext ${ }^{\circledR}$ Ultra $^{\text {TM }}$ directional RNA library prep kit for Illumina ${ }^{\circledR}$ (NEB, USA), according to the manufacturer's instructions. Next, DNA fragments were purified with AMPure XP (Beckman Coulter, Beverly, USA) and treated with $3 \mu$ lof USER enzyme (NEB, USA) at $37^{\circ} \mathrm{C}$ for $15 \mathrm{~min}$. DNA fragments were then amplified using Phusion High-Fidelity DNA polymerase, universal PCR primers, and index (X) primers. Finally, PCR products were purified using AMPure XP and their quality was evaluated using the Agilent Bioanalyzer 2100.

Index-coded samples were clustered on a cBot cluster generation system with a HiSeq. 4000 PE cluster kit (Illumina). Afterwards, DNA libraries were sequenced on the Illumina Hiseq 4000 platform.

Bioinformatics analyses. Clean reads were obtained from the raw data after removing adaptors, reads with an $\mathrm{N}$ ratio higher than $1 \%$, and low quality reads (with $>50 \%$ bases having Phred quality score $\leq 15$ ). Afterwards, clean reads were mapped to the reference genome (BioProject number in GenBank: PRJNA435474) using STAR 2.51. $\mathrm{b}^{17}$. HTSeq v0.6.0 $0^{18}$ was employed to calculate FPKM values (expected number of fragments per kilobase of transcript sequence per million base pairs sequenced) of each unigene. The relative expression levels of each gene were compared among different age groups using the DESeq2 ${ }^{19}$ in R (https://www.r-project.org). Pairwise comparisons were performed for each unigene between each two groups (e.g., juvenile vs. youth, juvenile vs. senior adult, etc.). Comparisons with $Q$ values $<0.05$ and $\mid \log _{2}$ FoldChange $>1$ were considered significantly differentially expressed.

Differentially expressed genes (DEGs) were mapped to the KEGG database (Kyoto Encyclopedia of Genes and Genomes) of Bos mutus (wild yak, database ID: T02919 in https://www.genome.jp) using ClusterProfiler3 ${ }^{20}$ to find out significantly enriched pathways. $\mathrm{P}$ values were adjusted using the $\mathrm{BH}$ method $^{21}$. To prevent a high false discovery rate (FDR) in multiple testing, $Q$ values were also estimated for FDR control ${ }^{22}$. The cut-off of adjusted $P$ values and $Q$ values was both set at 0.05 .

Real-time quantitative PCR. To verify the expression levels of DEGs calculated by FPKM, qPCR was performed. cDNA was synthesized from total RNA (the same RNA samples for Illumina sequencing) using a BioRT cDNA first strand synthesis kit (Bioer, Hangzhou, China) with the oligo(dT) primer. qPCR reactions were performed on a Line Gene9600 Plus qPCR machine (Bioer, Hangzhou, China) using BioEasy master mix (Bioer, Hangzhou, China). Each reaction was repeated three times. Three biological replicates were also performed for each age group. Primers used in the present study are listed in Supplementary Table S1. Glyceraldehyde phosphate dehydrogenase (GAPDH) was used as the internal control ${ }^{16,23}$. The relative expression level of each gene was calculated using the $2^{-\Delta \Delta C t}$ method ${ }^{24}$. Student's $t$ tests were performed to compare the relative expression level of each unigene between each two age groups. $\mathrm{P}<0.05$ was considered statistically significant. 


\begin{tabular}{|l|l|l|l|l|}
\hline & 6-month & 30 -month & 60-month & 90-month \\
\hline 6-month & - & 238 & 338 & 248 \\
\hline 30-month & 219 & - & 1 & 253 \\
\hline 60 -month & 115 & 0 & - & 374 \\
\hline 90-month & 35 & 21 & 13 & - \\
\hline
\end{tabular}

Table 1. Numbers of differentially expressed genes in gluteal and lung tissues of 6, 30, 60, and 90-month old yaks. Blow diagonal: lung tissue; Above diagonal: gluteus.

\section{Results and Discussion}

Illumina sequencing. The original sequencing files have been deposited in the National Center for Biotechnology Information (NCBI) database (bioproject number PRJNA512958). A total of 12 lung and 12 gluteus samples were successfully sequenced, generating $78.7 \mathrm{M}$ to $101.0 \mathrm{M}$ clean reads. The sequence data of every sample showed Q20 values higher than $96.7 \%$ and Q30 values higher than $92.0 \%$, suggesting that the results of transcriptome sequencing were technically qualified (Supplementary Table S2).

Differentially expressed genes. When the transcriptome profiles of the 30 - and 60 -month old yaks were compared, only one unigene was differentially expressed in gluteal tissues $(\mathrm{Q}<0.05$; Foldchange $>2)$ and no unigenes showed significant differences in lung tissues (Table 1).

Compared with 6-month old yaks, 219 and 115 DEGs in gluteal tissues and 238 and 338 DEGs in lung tissues were detected in 60- and 90-month old yaks, respectively $(\mathrm{Q}<0.05$; Foldchange $>2)$. Compared with 90-month old yaks, 21 and 13 DEGs in gluteal tissues and 253 and 374 DEGs in lung tissues were detected in 30- and 60 -month old yaks, respectively $(\mathrm{Q}<0.05$; Foldchange $>2$; Table 1$)$.

Six unigenes from the lung tissues and six from gluteal tissues were selected for qPCR to validate the expression levels calculated by FPKM values. Melting curves showed a single peak for all tested genes, suggesting that the PCR primers were specific. In the lung tissue five of the six unigenes tested were identified as being differentially expressed between the 6-month yaks and other age groups in the transcriptome analysis, using qPCR (Student's t-test, $\mathrm{P}<0.05$ ), while one (histone $\mathrm{H} 2 \mathrm{~B}$ ) was not (Student's t-test, $\mathrm{P}>0.05$ ). In the gluteal tissues, four of the six tested unigenes revealed similar changing trends among the four age groups between qPCR validation and Illumina sequencing (Pearson's correlation, $\mathrm{P}<0.05$ ). The other two, aquaporin $12(\mathrm{AQP} 12)$ and fibrinogen (FGB), displayed significant differences between 3- and 30-month old yaks (Student's t-test, $\mathrm{P}<0.05$ ) in the transcriptome analyses but no significant change (Student's t-test, $\mathrm{P}>0.05$ ) in the qPCR validation (Fig. 1). Overall, these results suggest that the transcriptome results are reliable.

Comparison of lung tissues between yaks of different ages. DEGs in lung tissues comparing 6-month and 30-month yaks, as well as 6-month and 60-month yaks, were separately subjected to KEGG enrichment analyses. The results were quite similar and were combined in one list, generating 34 KEGG pathways (Supplementary Table S3). Among the results, "ko05414 dilated cardiomyopathy" might respond to low oxygen conditions. Results related to immune function include "ko05340 primary immunodeficiency", "ko04662 B cell receptor signaling pathway", and "Ko05330 allograft rejection". Moreover, several typical regulating pathways ("ko04024 cAMP signaling pathway", "ko04064 NF-kappa B signaling pathway", and "ko04115 p53 signaling pathway") were also detected, which might function in signal transduction. Concurrently, comparisons between 30- and 90-month old yaks and between 60- and 90-month yaks were significantly enriched in three KEGG pathways, including "ko05322 systemic lupus erythematosus", "ko05034 alcoholism" and "ko05152 tuberculosis" (Supplementary Table S3).

To further explore changes in immune function during yak development, DEGs in immunity-related KEGG pathways were picked up. B-lymphocyte CD19 and CD79A antigens were significantly lower in 90-month yak growth compared with 60-month yaks (Student's t-test, $\mathrm{P}<0.05$ ), with change folds of 1.75 and 5.2 , respectively. CD79B antigen was significantly lower in the 90-month group than in the 30 -month group (Student's t-test, $\mathrm{P}<0.05 ; 1.83$ times; Fig. 2 and Supplementary Table S4). CD79a and CD79b antigens are components of the cell-surface B-cell receptor (BCR) and CD19 is a B cell surface molecule modifying signals generated through BCR. CD79A, CD79B, and CD19 are involved in recognition and subsequent signal transduction of pathogens and other foreign substances in B cells ${ }^{25,26}$. Downregulation of CD79A, CD79B, and CD19 in 90-month old yaks suggests that the B-cell function declined at 90 months compared to 30 - and 60-month animals.

Transferrin receptor expression is upregulated during $\mathrm{T}$ cell activation ${ }^{27}$, which is required for DNA synthesis and cell division ${ }^{28}$. In the present study, transferrin receptor levels were 2.59 and 2.67 times significantly lower in 90 -month old yaks than in 30- and 60-month old yaks, respectively (Student's t-test, $\mathrm{P}<0.05$, Fig. 2 and Supplementary Table S4), suggesting that activated $\mathrm{T}$ cell levels might be reduced in 90 -month old yaks, similar to those of B-cells.

Development, differentiation, and T and B lymphocyte function can be mediated by cytokines, such as complement factor $\mathrm{H}(\mathrm{CFH})$ not only plays a critical role in the homeostasis of the complement system in plasma but also functions as an important regulatory protein in innate immune system ${ }^{29}$. Compared with the 30 - and 60-month old groups, expression levels of CFH were 6.03 and 9.32 times significantly higher, and those of the transferrin receptor were 1.49 and 1.45 times significantly higher, at the 6-month stage, respectively (Student's t-test, all P values $<0.05$; Fig. 2 and Supplementary Table S4). Increased CFH and transferrin receptor expression levels suggested that immune functions might be more activated at 6-month stage compared with 30-month and 60 -month stages. Moreover, interleukin-1 (IL-1) could induce production of $\mathrm{CFH}^{30}$ and transferrin receptor ${ }^{31}$. In the present study, expression level of IL-1 was 3.31 and 2.04 times significantly higher at 6-month stage in 


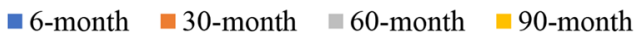

(A) Results of qPCR validation in lung

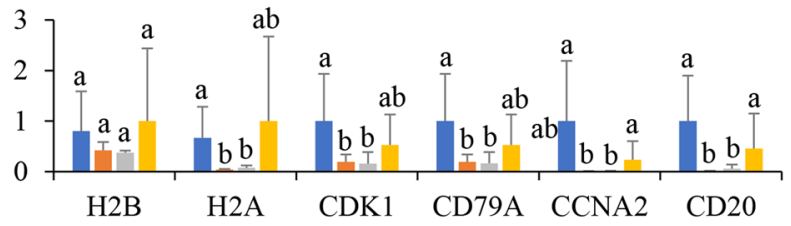

(B) Results based FPKM values in lung

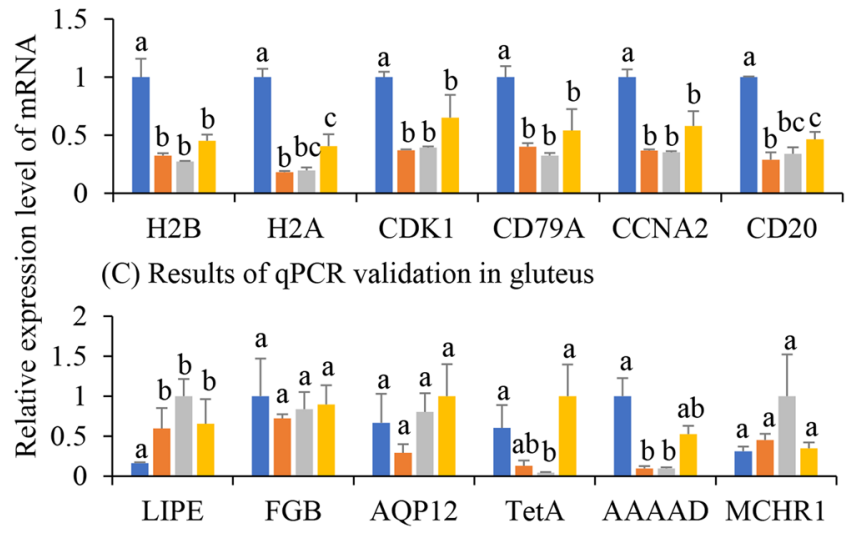

(D) Results based FPKM values in gluteus

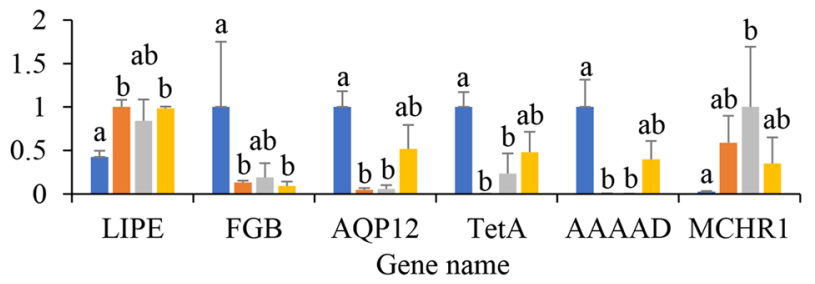

Figure 1. Real-time quantitative PCR validation of differentially expressed genes. H2B: histone H2B;

H2A: histone H2A; CDK1: cyclin-dependent kinase 1; CD79A: CD79A antigen; CCNA2: cyclin (A) CD20: B-lymphocyte antigen CD20; LIPE: Hormone-sensitive lipase; FGB: Fibrinogen; AQP12: Aquaporin 12; TetA: Tetracycline resistance protein; AAAAD: AAA + ATPase domain; MCHR1: Melanin-concentrating hormone 1. Student's t-tests were performed to determine differences in each unigene between age groups. Different letters above the bars indicated significant differences.

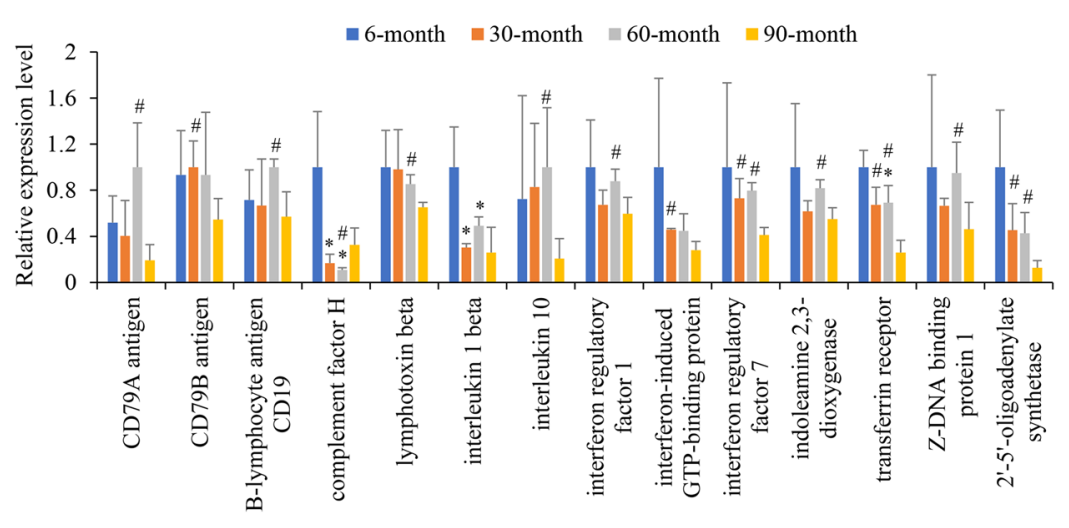

Figure 2. Relative expression levels of differentially expressed genes associated with immune function in lung tissue. All data were calculated based on the FPKM values and were normalized by defining the highest FPKM value among the four age groups as one. Errors donate standard deviation. Student's t-tests were performed to determine differences in each unigene between age groups. *Significantly different from 6-month old group $(\mathrm{P}<0.05)$. "Significantly different from 90 -month $(\mathrm{P}<0.05)$. 
comparison to 30-month and 60-month stage, respectively (Student's t-test, both P values $<0.05$ ), displaying similar changing trends with CFH and transferrin receptor (Pearson's correlation, $\mathrm{P}<0.05$; Fig. 2 and Supplementary Table S4). These results suggested that higher activation status of immune function in 6-month yak might be regulated by IL-1. Interferons (IFNs) are mainly synthesized and released by helper CD4 T lymphocytes, monocytes, macrophages, and endothelial cells $\mathrm{s}^{32}$. These cytokines regulate immune and inflammatory response to infections ${ }^{33,34}$. In the present study, IFN regulatory factor (IRF) 1 levels were 1.5 times significantly lower in 90-month old yaks than in 60-month old animals (Student's t-test, $\mathrm{P}<0.05$ ). IRF-7 levels were 1.77 and 1.93 times significantly lower in 90-month-olds than in 30- and 60-month-olds, respectively (Student's t-test, both $\mathrm{P}<0.05)$. IFN-induced GTP-binding protein was 1.64 times significantly lower in 90 -month old yaks than in 30 -month yaks (Student's t-test, $\mathrm{P}<0.05$ ). Moreover, $2^{\prime}-5^{\prime}$-oligoadenylate synthetase (OAS2), which is stimulated by IFN ${ }^{35}$, also displayed significantly lower levels in the 90 -month group than in the with 30 - and 60-month groups, with change folds of 3.58 and 3.38, respectively (Student's t-test, all $\mathrm{P}<0.05$ ). An upstream activator of IRF, Z-DNA binding protein 1 (ZBP1), which can detect DNA from viral, bacterial, or even host origin ${ }^{36}$, was 1.31 times significantly lower in the 90 -month yaks than in the 60 -month yaks (Student's t-test, $\mathrm{P}<0.05$; Fig. 2 and Supplementary Table S4). These results indicate that IFN levels might be reduced in 90 -month old yaks compared with 30- and 60-month old yaks. The expression level of IL-10 was 4.83 times lower in the 90-month than in the 60-month group (Student's t-test, $\mathrm{P}<0.05$ ). Furthermore, indoleamine 2,3-dioxygenase (IDO), which has been reported to enhance production of interleukin $10^{37}$, showed a 1.49 times significantly lower expression level at 90-months than at 60-months (Student's t-test, $\mathrm{P}<0.05$; Fig. 2 and Supplementary Table S4). Changes in the levels of these two proteins indicate that IL-10 is less expressed in 90-month old yaks compared to 60-month old yaks. In addition, the cytokine lymphotoxin showed significantly lower levels (1.31 times) in the 90-month than the 60 -month group (Student's t-test, $\mathrm{P}<0.05$ ). IFN regulates $\mathrm{B}$-cell function in humans $\mathrm{s}^{38}$ and IL-10 $0^{39}$ and lymphotoxin $^{40}$ act as autocrine growth or activation factors for B lymphocytes. Thus, it is possible that reduced B-cell function in 90-month old yaks is probably attributed to decreased levels of IFN, IL-10 and lymphotoxin.

Activation of immune functions in 6-month yaks might be attributed to their immature physiological development. First, high-altitude environments experience strong UV radiation, low oxygen, and low temperatures. These factors can negatively affect the immune system and make animals more susceptible to infections ${ }^{41}$. As reported previously, the dermis thickness is lower in juvenile yak than adults ${ }^{10}$. Thus, 6 -month old yaks should be more susceptible to low oxygen, low temperature, and high UV exposure. Increased immune activation could help juvenile yaks resist the dangers of a high-altitude environment. Second, the lungs are weaker in juvenile yaks than in adults. Enhanced immune function in juveniles could prevent acute mountain sickness, high altitude pulmonary edema, and high altitude cerebral edema ${ }^{42}$.

Moreover, unigenes related to the cell cycle are also differentially expressed in lung tissues among different aged yaks. Cyclins regulate the cell cycle by activating cyclin-dependent kinases (CDKs) ${ }^{43}$. Cyclin-dependent kinase regulatory subunit 1 (CKS1) regulates the cell cycle to increase cell numbers ${ }^{44}$. Mitotic checkpoint serine/ threonine-protein kinase (Bub1) and condensin complex subunit 3 are required for chromosome congression, assembly, and segregation during mitosis and meiosis ${ }^{45,46}$. Overexpression of the transcription factor E2F2 in nonproliferating rabbit corneal endothelial cells has been shown to induce cell cycle progression without prompting significant apoptosis ${ }^{47}$. In the present study, CDK1 levels were 1.96 and 1.70 times significantly more highly expressed in 6-month old yaks compared with 30-month and 60-month, respectively (Student's t-test, both $\mathrm{P}<0.05)$. Cyclin A levels were 1.67 and 1.93 times significantly lower in 90 -month old lung tissues than in those from 30- and 60-month animals, respectively (Student's t-test, P $<0.05$ ). CKS1 and E2F2 levels were 2.33 and 2.63 times significantly lower at 90 -months than at 30 -months (Student's t-test, both $\mathrm{P}<0.05$ ). BUB1 showed 2.31 times significantly higher expression at 6 -months than at 30 -months (Student's t-test, $\mathrm{P}<0.05$; Fig. 3 and Supplementary Table S4). These results suggest that the cell cycle was promoted at 6-months but was decreased at 90-months, in comparison with 30- and 60-month yak lung tissues. Thus, the growth of lung tissues should be the fastest at 6 -month of age, following by 30 - and 60 -months, and reaching the slowest rate of growth at 90-months. Moreover, 12 DEGs encoding histone showed significantly higher expression levels in 6-month old yak lung tissues than in 30 -month tissues (Student's t-test, $\mathrm{P}<0.05$ ). Three histone unigenes were significantly lower at 90-months than at 60-months (Student's t-test, P $<0.05$; Fig. 3 and Supplementary Table S4). Histones are the chief protein component of chromatin, and must accumulate before cell division. The changes in histone expression levels also support our hypothesis in relation to cell cycle.

Comparison of gluteal tissues between yaks of different ages. In gluteal tissues, DEGs comparisons between 6- and 30-months and between 6- and 60-months were separately subjected to KEGG enrichment analyses. The as-obtained pathways were combined into one list. Finally, a total of four KEGG pathways were significantly over-represented when comparing 6- and 30-month yaks, and between 6- and 60-month yaks. Comparisons between 30 - and 90-month and between 60 - and 90-month gluteal tissues showed 26 significantly enriched KEGG pathways. Among them, 14 were functionally related to the immune system, including "ko04650 natural killer cell mediated cytotoxicity", "ko04672 intestinal immune network for IgA production", "ko04060 cytokine-cytokine receptor interaction", and "ko05340 primary immunodeficiency" (Supplementary Table S5).

Compared with the 6-month group, expression levels of macrophage scavenger receptor 1 were 1.55 and 1.31 times lower, CD48 levels were 1.98 and 1.91 times lower, CD86 levels were 1.65 and 1.47 times lower, T-cell receptor beta chain $\mathrm{V}$ region (BmuPB011165) levels were 1.49 and 1.43 times lower, complement factor $\mathrm{H}$ levels were 5.75 and 14.13 times lower, and interferon levels were 2.20 and 1.73 times lower than the same levels at 30and 60-months, respectively (all the comparisons were statistically significant, Student's t-test, $\mathrm{P}$ values $<0.05$; Fig. 4 and Supplementary Table S6). These results indicate that immune function at 6-months is more activated than at 30- and 60-months, which could be due to the immature developmental status and increased need to resist high-altitude environments at 6-months of age, similar to the results observed in lung tissues. Moreover, 


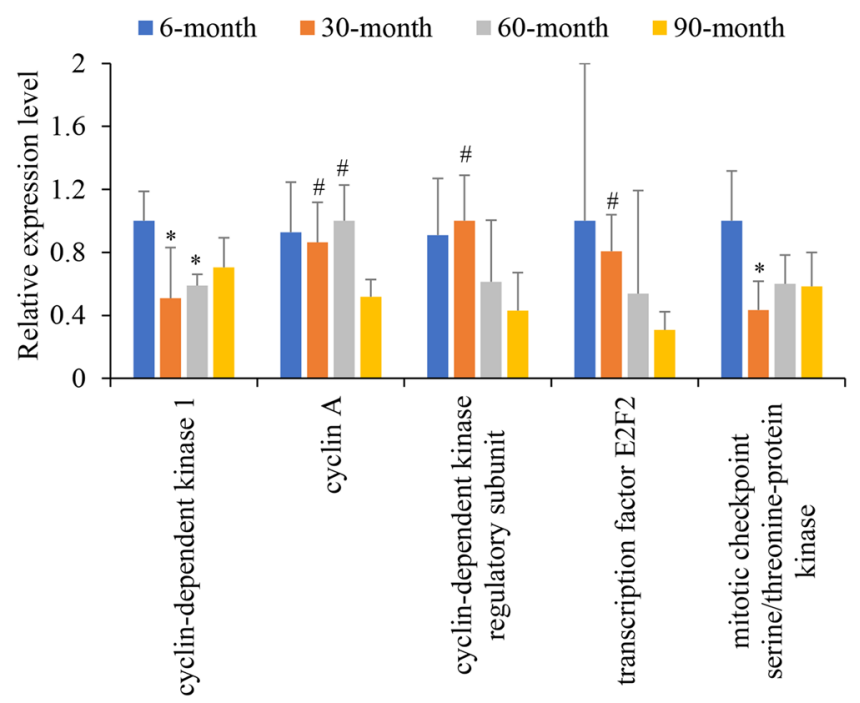

Figure 3. Relative expression levels of differentially expressed genes associated with the cell cycle in lung tissue. All data were calculated based on the FPKM values and were normalized by defining the highest FPKM value among the four age groups as one. Errors donate standard deviation. Student's t-tests were performed to determine differences in each unigene between age groups. *Significantly different from 6-month old group $(\mathrm{P}<0.05)$. "Significantly different from 90 -month old group $(\mathrm{P}<0.05)$.

interleukin 2 receptor (IL-2R) beta levels were 1.74 and 1.41 times significantly higher and IL-2R gamma levels were 1.46 and 1.53 times significantly higher at 6-months than at 30- and 60-months, respectively (Student's $\mathrm{t}$-test, $\mathrm{P}<0.05$ ). As reported, IL-2R is responsible for transducing signals from IL-2 and T-cell dependent activity $^{48}$. Thus, it is possible that the activation of immune factors in 6-month old muscle tissues is regulated by IL- 2 .

Compared with 30- and 60-month yaks, the T-cell surface glycoprotein CD3 delta chain levels were 1.46 and 1.40 times higher, CD3 gamma chain levels were 1.50 and 1.44 times higher, lymphocyte cell-specific protein tyrosine kinase levels were 1.43 and 1.37 times higher, T-cell receptor beta chain V region (BmuPB011166) levels were 1.30 and 1.47 times higher, and interferon levels were 1.78 and 1.42 times higher at 90 -months, respectively (all the comparisons were statistically significant, Student's t-test, $\mathrm{P}$ values $<0.05$ ). These results indicate that immune activation in muscle tissues is higher in 90-month old yaks than in 30- and 60-month animals. In older yaks, muscles might be more vulnerable and muscle injuries would induce immune activation ${ }^{49}$. To test this hypothesis, genes associated with muscle tenderness, injury, and regeneration were investigated. First, at the 90 -month stage, four unigenes encoding collagen/elastin were significantly lower than those at the 30-month stage (change fold ranged from 1.63 to 2.01) and three collagen/elastin unigenes were significantly depressed compared with levels at 60-months (change fold ranged from 1.69 to 2.01) (Student's t-test, all P values $<0.05$; Fig. 4 and Supplementary Table S6). Muscle tenderness has been positively correlated with intramuscular collagen content in bovines ${ }^{50}$. Our results suggest that 90 -month old yaks might suffer muscle injuries more readily, due to low muscle tenderness, than do 30- and 60-month old yaks. Second, four unigenes encoding the C-C chemokine receptor were significantly higher in 90 -month than in 30-month yaks (change folds ranged from 1.54 to 1.82 , Student's t-test, all $\mathrm{P}$ values $<0.05$ ), and the $\mathrm{C}-\mathrm{C}$ chemokine receptor type 5 level was 1.41 times higher in 90-month than in 60-month old yaks (Student's t-test, $\mathrm{P}<0.05$ ). The unigene BmuPB014660, encoding tumor necrosis factor receptor superfamily member 5, was 1.41 times significantly higher than in 60 -month old yaks (Student's t-test, $\mathrm{P}<0.05$ ). The expression level of integrin alpha $\mathrm{M}$ was 1.94 times significantly higher at 90 -months than at 60-months (Student's t-test, $\mathrm{P}<0.05$; Supplementary Table S6). C-C chemokine receptor ${ }^{51,52}$, tumor necrosis factor (TNF) $)^{53,54}$, and integrin ${ }^{55}$ have been reported to participate in muscle injury responses and the subsequent regeneration of new tissues. Thus, overexpression of these unigenes at 90 -months of age suggests that 90 -month old yaks might undergo more muscle injuries. Furthermore, the ras-related C3 botulinum toxin substrate 2 level was 1.29 times significantly higher in 90-month than 60-month old yaks (Student's t-test, $\mathrm{P}<0.05)$ The ras superfamily regulates cell differentiation, proliferation, and cytoskeletal organization ${ }^{56}$. The observed high expression of ras-related C3 botulinum toxin substrate 2 supports the evidence that increased regeneration of new muscle tissues might take place in 90 -month old yaks. Overall, these results indicated that muscle tissue at 90-months of age was more highly activated than at 30- and 60-months of age, which is probably due to the lower muscle tenderness and more severe injuries at 90 -months.

\section{Conclusions}

The present study reveals that immune activation status differs between yaks of different ages. In lung tissues, immune function was more activated at 6-months of age and less activated at 90-months, compared with activation at 30- and 60-months. In gluteal tissues, immune function was more activated at 6-and 90-months than at 30- and 60-months. This activation of the immune system could increase the resistance of juvenile yaks to 


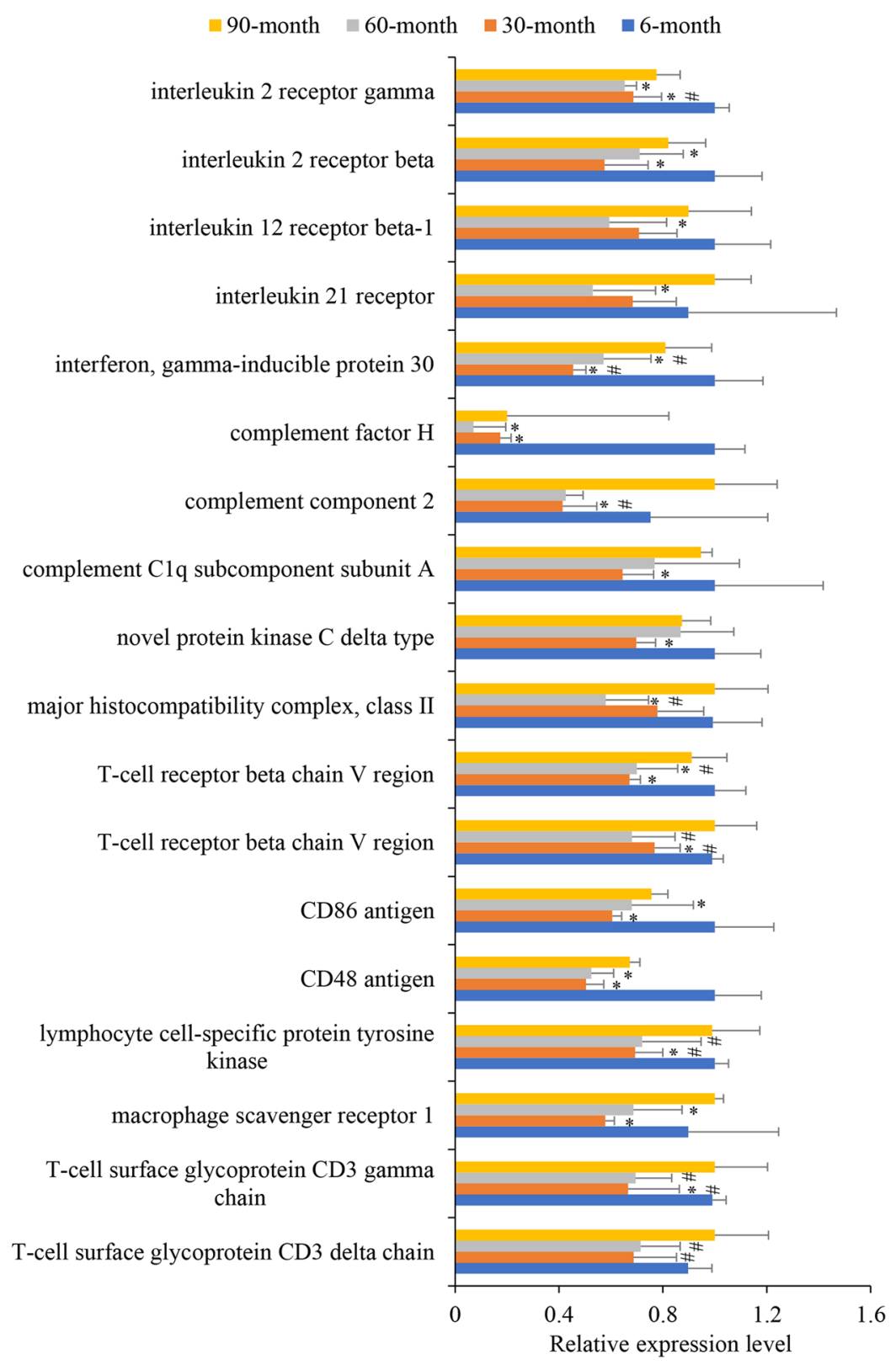

Figure 4. Relative expression levels of differentially expressed genes associated with immune function in gluteal tissue. All data were calculated based on the FPKM values and were normalized by defining the highest FPKM value among the four age groups as one. Errors donate standard deviation. Student's t-tests were performed to determine differences in each unigene between age groups. *Significantly different from 6-month old group $(\mathrm{P}<0.05)$. "Significantly different from 90 -month old group $(\mathrm{P}<0.05)$.

high-altitude environments. Enhanced immune function in senior yak gluteal tissues might be a response to muscle injuries resultant from low muscle tenderness.

\section{Data Availability}

The original sequencing files have been deposited in the National Center for Biotechnology Information (NCBI) with the bioproject number PRJNA512958.

\section{References}

1. Long, R. J., Apori, S. O., Castro, F. B. \& Ørskov, E. R. Feed value of native forages of the Tibetan Plateau of China. Animal Feed Science \& Technology 80, 101-113(1999).

2. Guan, J. et al. Comparative analysis of the microRNA transcriptome between yak and cattle provides insight into high-altitude adaptation. Peerj 5, e3959 (2017).

3. Durmowicz, A. G., Hofmeister, S., Kadyraliev, T. K., Aldashev, A. A. \& Stenmark, K. R. Functional and structural adaptation of the yak pulmonary circulation to residence at high altitude. Journal of Applied Physiology 74, 2276-2285 (1993). 
4. Dolt, K. S. et al. cDNA cloning, gene organization and variant specific expression of HIF-1 alpha in high altitude yak (Bos grunniens). Gene 386, 73-80 (2007).

5. Wang, H. et al. A comparative study on urinary purine derivative excretion of yak (Bos grunniens), cattle (Bos taurus), and crossbred (Bos taurus $\times$ Bos grunniens) in the Qinghai-Tibetan plateau, China. Journal of Animal Science 87, 2355 (2009).

6. Wang, H. et al. Comparison of Nitrogen Metabolism in Yak (Bos grunniens) and Indigenous Cattle (Bos taurus) on the QinghaiTibetan Plateau. Asian Australasian Journal of Animal Sciences 24, 766-773 (2011).

7. Qiu, Q. et al. The yak genome and adaptation to life at high altitude. Nature Genetics 44, 946-949 (2012).

8. Lan, D. L. et al. Transcriptome profile and unique genetic evolution of positively selected genes in yak lungs. Genetica 146, 1-10 (2017).

9. Wang, K. et al. Different gene expressions between cattle and yak provide insights into high-altitude adaptation. Animal Genetics 47, 28-35 (2016).

10. Chuan, Y. U., Cui, Y., Yang, X. \& Yue, J. Thickness changes of skin in different age yak (Bos grunniens). Journal of Gansu Agricultural University (2017).

11. Jun-Feng, H. E., Si-Jiu, Y. U. \& Cui, Y. Characteristics of Lung Structure in Different Age Plateau Yak. Chinese Journal of Animal \& Veterinary Sciences 40, 748-755 (2009).

12. Zhang, Q. et al. Age-related changes in the morphology and protein expression of the thymus of healthy yaks (Bos grunniens). American Journal of Veterinary Research 77, 567 (2016).

13. Li, M. et al. Comparative iTRAQ proteomics revealed proteins associated with horn development in yak. Proteome Science 16, 14 (2018).

14. Zhao, H. et al. Comparative antennal transcriptome of Apis cerana cerana from four developmental stages. Gene 660, 102-108 (2018).

15. Yang, H. et al. Transcriptome analysis in different developmental stages of Batocera horsfieldi (Coleoptera: Cerambycidae) and comparison of candidate olfactory genes. Plos One 13, e0192730 (2018).

16. Xin, J. W. et al. Transcriptome profiles revealed the mechanisms underlying the adaptation of yak to high-altitude environments. Scientific Reports 9, 7558 (2019).

17. Dobin, A. et al. Star: ultrafast universal rna-seq aligner. Bioinformatics 29, 15-21 (2013).

18. Anders, S., Pyl, P. T. \& Huber, W. HTseq-a python framework to work with high-throughput sequencing data. Bioinformatics 31, $166-169$ (2015).

19. Love, M. I., Huber, W. \& Anders, S. Moderated estimation of fold change and dispersion for RNA-seq data with DESeq. 2. Genome Biology 15, 550 (2014).

20. Luo, W. \& Brouwer, C. Pathview: An R/Bioconductor package for pathway-based data integration and visualization. Bioinformatics 29, 1830-1831 (2013).

21. Benjamini, Y. \& Hochberg, Y. Controlling the false discovery rate: a practical and powerful approach to multiple testing. Journal of the Royal Statistical Society. Series B 57, 289-300 (1995).

22. Storey, J. D. A direct approach to false discovery rates. Journal of the Royal Statistical Society. Series B 64, 479-498 (2002).

23. Varshney, N. et al. Selection of suitable reference genes for quantitative gene expression studies in milk somatic cells of lactating cows (Bos indicus). Journal of Dairy Science 95, 2935-2945 (2012).

24. Livak, K. J. \& Schmittgen, T. D. Analysis of Relative Gene Expression Data Using Real-Time Quantitative PCR and the $2^{-\Delta \Delta C T}$ Method. Methods 25, 402-408 (2001).

25. Chu, P. G. \& Arber, D. A. CD79: a review. Applied Immunohistochemistry \& Molecular Morphology 9, 97-106 (2001).

26. Wagle, N. M., Kim, J. H. \& Pierce, S. K. CD19 regulates B cell antigen receptor-mediated MHC class II antigen processing. Vaccine 18, 376-386 (1999).

27. Bayer, A. L., Baliga, P. \& Woodward, J. E. Transferrin receptor in T cell activation and transplantation. Journal of Leukocyte Biology 64, 19-24 (1998).

28. Neckers, L. M. \& Cossman, J. Transferrin Receptor Induction in Mitogen-Stimulated Human T Lymphocytes Is Required for DNA Synthesis and Cell Division and Is Regulated by Interleukin-2 (TCGF). In: Goldstein A. L. (eds) Thymic Hormones and Lymphokines. GWUMC Department of Biochemistry Annual Spring Symposia. Springer, Boston, MA (1984)

29. Rodríguez de Córdoba, S. et al. The human complement factor $\mathrm{H}$ : functional roles, genetic variations and disease associations. Molecular Immunology 41, 355-367 (2004).

30. Minta, J. O. Regulation of complement factor $\mathrm{H}$ synthesis in U-937 cells by phorbol myristate acetate, lipopolysaccharide, and IL-1. The Journal of Immunology 141, 1630-1635 (1988).

31. Tsuji, Y., Miller, L. L., Miller, S. C., Torti, S. V. \& Torti, F. M. Tumor necrosis factor-alpha and interleukin 1-alpha regulate transferrin receptor in human diploid fibroblasts. Relationship to the induction of ferritin heavy chain. Journal of Biological Chemistry 266, 7257-7261 (1991).

32. Kaiser, P. R. L., Avery, S. \& Balu, S. Evolution of the interleukins [review]. Developmental \& Comparative Immunology 28, 375-394 (2004).

33. Brocker, C., Thompson, D., Matsumoto, A., Nebert, D. W. \& Vasiliou, V. Evolutionary divergence and functions of the human interleukin (IL) gene family. Human Genomics 5, 30-55 (2010).

34. De Andrea, M., Ravera, R., Gioia, D., Gariglio, M. \& Landolfo, S. The interferon system: an overview. European Journal of Paediatric Neurology 6, A41-A46 (2002).

35. Johnson, G. A. et al. Effects of the estrous cycle, pregnancy, and interferon tau on $2^{\prime}, 5^{\prime}$-oligoadenylate synthetase expression in the ovine uterus1. Biology of Reproduction 64, 1392-1399 (2001).

36. Takaoka, A. et al. DAI (DLM-1/ZBP1) is a cytosolic DNA sensor and an activator of innate immune response. Nature 448, 501-505 (2007).

37. van der Sluijs, K. F. et al. Influenza-induced expression of indoleamine 2,3-dioxygenase enhances interleukin-10 production and bacterial outgrowth during secondary pneumococcal pneumonia. The Journal of Infectious Diseases 193, 214-222 (2006).

38. Punnonen, J. \& Viljanen, M. K. Interferon- $\alpha$ and interferon- $\gamma$ as regulators of human adult and newborn B-cell function. International Journal of Immunopharmacology 11, 717-723 (1989).

39. Burdin, N., Rousset, F. \& Banchereau, J. B-cell-derived IL-10: production and function. Methods 11, 98-111 (1997).

40. Tumanov, A. V. et al. T cell-derived lymphotoxin regulates liver regeneration. Gastroenterology 136, 694-704 (2009).

41. Mishra, K. P. \& Ganju, L. Influence of high altitude exposure on the immune system: a review. Immunological Investigations 39, 219-234 (2010).

42. Khanna, K., Mishra, K. P., Ganju, L., Kumar, B. \& Singh, S. B. High-Altitude-Induced alterations in Gut-Immune Axis: A review. International Reviews of Immunology 37, 1 (2017).

43. Bloom, J. \& Cross, F. R. Multiple levels of cyclin specificity in cell-cycle control. Nature Reviews Molecular Cell Biology 8, 149-160 (2007).

44. Liu, C. Y., Zhao, W. L., Wang, J. X. \& Zhao, X. F. Cyclin-dependent kinase regulatory subunit 1 promotes cell proliferation by insulin regulation. Cell Cycle 14, 3045-3057 (2015).

45. Hirano, T. Condensin-based chromosome organization from bacteria to vertebrates. Cell 164, 847-857 (2016).

46. Johnson, V. L., Scott, M. I. F., Holt, S. V., Hussein, D. \& Taylor, S. S. Bub1 is required for kinetochore localization of BubR1, Cenp-E, Cenp-F and Mad2, and chromosome congression. Journal of Cell Science 117, 1577-1589 (2004). 
47. Joyce, N. C. Effect of overexpressing the transcription factor E2F2 on cell cycle progression in rabbit corneal endothelial cells. Investigative Ophthalmology \& Visual Science 45, 1340-1348 (2004).

48. Malek, T. R. \& Castro, I. Interleukin-2 receptor signaling: at the interface between tolerance and immunity. Immunity 33, 153-165 (2010).

49. Tidball, J. G. Interactions between muscle and the immune system during modified musculoskeletal loading. Clinical Orthopaedics \& Related Research 403, S100-S109 (2002).

50. Cross, H. R., Carpenter, Z. L. \& Smith, G. C. Effects of intramuscular collagen and elastin on bovine muscle tenderness. Journal of Food Science 38, 998-1003 (1973).

51. Yahiaoui, L., Gvozdic, D., Danialou, G., Mack, M. \& Petrof, B. J. CC family chemokines directly regulate myoblast responses to skeletal muscle injury. The Journal of Physiology 586, 3991-4004 (2008).

52. Schecter, A. D. et al. Human vascular smooth muscle cells possess functional CCR5. Journal of Biological Chemistry 275, 5466-5471 (2000).

53. Warren, G. L. et al. Physiological role of tumor necrosis factor a in traumatic muscle injury. The FASEB Journal 16, 1630-1632 (2002).

54. Gaines, G. C. et al. Attenuation of skeletal muscle ischemia/reperfusion injury by inhibition of tumor necrosis factor. Journal of Vascular Surgery 29, 370-376 (1999).

55. Kaariainen, M. et al. Integrin and dystrophin associated adhesion protein complexes during regeneration of shearing-type muscle injury. Neuromuscular Disorders 10, 121-132 (2000).

56. Alberts, B., Johnson, A. \& Lewis, J. Molecular Biology of the Cell. Garland Science. pp. 877 (2002).

\section{Acknowledgements}

This work was supported by the Ring-fenced Funding of the Finance Department of Tibet Autonomous Region and the National Beef Cattle and Yak Industrial Technology System (No: CARS-37).

\section{Author Contributions}

J.W.X., Q.M.J. and J.C.Z. designed the experiments. J.W.X., Z.X.C., C.F.Z., Q.Z., Y.Z. and H.W.C. collected the samples, sequenced the transcriptomes, and analyzed the data. J.W.X. drafted the manuscript and all other authors revised it.

\section{Additional Information}

Supplementary information accompanies this paper at https://doi.org/10.1038/s41598-019-50618-x.

Competing Interests: The authors declare no competing interests.

Publisher's note Springer Nature remains neutral with regard to jurisdictional claims in published maps and institutional affiliations.

(c) (i) Open Access This article is licensed under a Creative Commons Attribution 4.0 International

License, which permits use, sharing, adaptation, distribution and reproduction in any medium or format, as long as you give appropriate credit to the original author(s) and the source, provide a link to the Creative Commons license, and indicate if changes were made. The images or other third party material in this article are included in the article's Creative Commons license, unless indicated otherwise in a credit line to the material. If material is not included in the article's Creative Commons license and your intended use is not permitted by statutory regulation or exceeds the permitted use, you will need to obtain permission directly from the copyright holder. To view a copy of this license, visit http://creativecommons.org/licenses/by/4.0/.

(c) The Author(s) 2019 